\title{
STABILITY ANALYSIS OF A DELAYED FRACTIONAL ORDER SIRS EPIDEMIC MODEL WITH NONLINEAR INCIDENCE RATE
}

\author{
Mouhcine Naim ${ }^{1} \S$, Fouad Lahmidi ${ }^{2}$, Abdelwahed Namir ${ }^{3}$ \\ ${ }^{1,2,3}$ Department of Mathematics and Computer Science \\ Faculty of Sciences Ben M'sik \\ Hassan II University, P.O. Box 7955 \\ Sidi Othman, Casablanca, MOROCCO
}

\begin{abstract}
In this paper, we study the stability of a fractional order SIRS epidemic model with nonlinear incidence rate and time delay, where the fractional derivative is defined in the Caputo sense. The delay is introduced into the model in order to modeled the incubation period. Using the stability analysis of delayed fractional order systems, we prove that the disease-free equilibrium is locally asymptotically stable when the basic reproduction number $R_{0}<1$. Also, we show that if $R_{0}>1$, the endemic equilibrium is locally asymptotically stable.
\end{abstract}

AMS Subject Classification: $34 \mathrm{~A} 08,37 \mathrm{C} 75,92 \mathrm{D} 30$

Key Words: SIRS epidemic model, fractional order, time delay, stability

\section{Introduction}

Epidemiology is the study of the spread of diseases in human populations and the factors that are responsible for or contribute to their occurrence. Consequently, it has been investigated by several researchers through study of the dynamical behavior of infectious diseases by mathematical models (see, e.g., $[1,2,3,4,5,6,7,8])$.

Received: March 6, 2019

(c) 2019 Academic Publications

${ }^{\S}$ Correspondence author 
Fractional calculus is the field of mathematical analysis aiming at the investigation of integrals and derivatives of arbitrary (non integer) orders. The main advantage of fractional order derivative in comparison in integer order is that fractional order derivative can be describe the memory and hereditary effects in various substances. Therefore, many applied researchers have treat many real processes using the fractional derivative such as $P I^{\lambda} D^{\mu}$ controller [9], human root dentin [10], botanical electrical impedances [11], viscoelasticity of cancellous bone [12], financial processes [13], epidemic model [14], and so on.

Due to the memory effect which is has an important role on the spread of an infectious disease, many investigators have started to study the epidemic models using the fractional derivative, see, e.g., $[15,16,17,18,19]$. In this work, we propose the following delayed fractional order SIRS epidemic model with nonlinear incidence rate

$$
\left\{\begin{aligned}
D^{\theta} S(t) & =\Lambda-\mu S(t)-\frac{\beta S(t) I(t)}{1+\alpha_{1} S(t)+\alpha_{2} I(t)+\alpha_{3} S(t) I(t)}+\gamma R(t), \\
D^{\theta} I(t) & =\frac{\beta S(t-\tau) I(t-\tau)}{1+\alpha_{1} S(t-\tau)+\alpha_{2} I(t-\tau)+\alpha_{3} S(t-\tau) I(t-\tau)}-(\mu+\alpha+d) I(t), \\
D^{\theta} R(t) & =\alpha I(t)-(\mu+\gamma) R(t),
\end{aligned}\right.
$$

where $\theta \in(0,1]$ is the order of the fractional derivative. $S(t), I(t)$ and $R(t)$ are the numbers of susceptible, infective and recovered individuals at time $t$, respectively. $\Lambda>0$ is the recruitment rate of susceptibles, $\mu>0$ is the natural death rate of the population, $d>0$ is the death rate due to disease, $\alpha>0$ is the recovery rate of the infective individuals and $\gamma \geq 0$ is the rate at which recovered individuals lose immunity and return to susceptible class. The incidence rate of disease is modeled by the specific functional response $\frac{\beta S I}{1+\alpha_{1} S+\alpha_{2} I+\alpha_{3} S I}$ presented by hattaf et al. [20], where $\beta>0$ is the contact transmission coefficient, which measures the infection force of the disease and $\alpha_{1}, \alpha_{2}, \alpha_{3} \geq 0$ are saturation factors measuring the psychological or inhibitory effect. $\tau \geq 0$ is the time delay, and it represents the incubation period, i.e., the time during which an infected individual will become infectious. When $\gamma=0$, the individuals recovered acquired permanent immunity, and (1) reduces to an SIR model.

The purpose of this paper is to study the influences of the fractional order and time delay on the stability of the equilibria of the model (1). So, the paper is organized as follows. In the next section, some definitions and lemmas are presented. We discuss the existence and the stability of the equilibria of model (1) in Section 3. The paper ends with a conclusion in Section 4. 


\section{Preliminaries}

The fractional order derivative used in model (1) is in the sense of Caputo definition, which is a modification of the Riemann-Liouville definition, and has the advantage that the initial values for fractional differential equations with Caputo derivative take the same form as that for integer order differential equations. Also, another advantage of this definition is that the Caputo derivative of a constant is zero.

The Riemann-Liouville fractional integral and Caputo fractional derivative are defined respectively as follows.

Definition 1. ([21, 22]) The Riemann-Liouville fractional integral of order $\theta>0$ for an integrable function $f: \mathbb{R}_{+} \mapsto \mathbb{R}$ is defined as

$$
I^{\theta} f(t)=\frac{1}{\Gamma(\theta)} \int_{0}^{t}(t-s)^{\theta-1} f(s) d s
$$

where $\Gamma$ is the gamma function

$$
\Gamma(\theta)=\int_{0}^{\infty} t^{\theta-1} e^{-t} d t
$$

Definition 2. ([21, 22]) The Caputo fractional derivative of order $\theta>0$ for a function $f \in \mathcal{C}^{n}\left(\mathbb{R}_{+}, \mathbb{R}\right)$ is defined as

$$
D^{\theta} f(t)=I^{n-\theta}\left[f^{(n)}(t)\right]=\frac{1}{\Gamma(n-\theta)} \int_{0}^{t}(t-s)^{n-\theta-1} f^{(n)}(s) d s,
$$

where $n$ is a positive integer such that $\theta \in(n-1, n]$. In particular, when $\theta \in(0,1]$, one has

$$
D^{\theta} f(t)=\frac{1}{\Gamma(1-\theta)} \int_{0}^{t}(t-s)^{-\theta} f^{\prime}(s) d s .
$$

Consider the following linear delayed fractional differential system

$$
D^{\theta} x(t)=A x(t)+B x(t-\tau), \quad t \geq 0,
$$

where $\theta \in(0,1], x(t) \in \mathbb{R}^{n}, A, B \in \mathbb{R}^{n \times n}$ and $\tau \geq 0$. The characteristic equation of system (2) is

$$
\Delta(s)=\operatorname{det}\left(s^{\theta} I_{n}-A-B e^{-s \tau}\right)=0 .
$$


If $\tau=0$, system (2) can be expressed as

$$
D^{\theta} x(t)=M x(t)
$$

where the coefficient matrix $M=A+B$.

In the case of $A=0$, Deng et al. in [23] obtained the following two stability results.

Lemma 3. If all the roots of the characteristic equation $\Delta(s)=0$ have negative real parts, then the zero solution of system (2) is Lyapunov globally asymptotically stable.

Lemma 4. If all the eigenvalues $\lambda$ of $B$ satisfy $|\arg (\lambda)|>\frac{\theta \pi}{2}$ and the characteristic equation $\Delta(s)=0$ has no purely imaginary roots for any $\tau>0$, then the zero solution of system (2) is Lyapunov globally asymptotically stable.

If $A \neq 0$, according to [24], we have the following result.

Lemma 5. If all the eigenvalues $\lambda$ of $M$ satisfy $|\arg (\lambda)|>\frac{\pi}{2}$ and the characteristic equation $\Delta(s)=0$ has no purely imaginary roots for any $\tau>0$, then the zero solution of system (2) is Lyapunov globally asymptotically stable.

Remark 6. If $A \neq 0$, the stability of system (2) is not guaranteed under conditions that the eigenvalues of $M$ are satisfied $|\arg (\lambda)|>\frac{\theta \pi}{2}$. In fact, when the eigenvalues of $M$ are satisfied $\frac{\theta \pi}{2}<|\arg (\lambda)| \leq \frac{\pi}{2}$, and the characteristic equation $\Delta(s)=0$ has no purely imaginary roots for any $\tau>0$, the zero solution has unstable situation (see Section 5 in [24]).

Remark 7. To study the local asymptotic stability of equilibria of nonlinear fractional order systems with time delay, we investigate the stability of the linearized systems of such nonlinear systems around these equilibria based on the previous lemmas.

\section{Stability analysis}

In this section, we discuss the existence and the local stability of the equilibria of system (1). In this sense, we define the basic reproduction number of model 
(1) as follows

$$
R_{0}=\frac{\beta \Lambda}{\left(\mu+\alpha_{1} \Lambda\right)(\mu+\alpha+d)},
$$

which represents the average number of secondary infections produced by one infected individual in a completely susceptible population [25].

The equilibria of system (1) satisfy the following equations

$$
\begin{aligned}
\Lambda-\mu S-\frac{\beta S I}{1+\alpha_{1} S+\alpha_{2} I+\alpha_{3} S I}+\gamma R & =0, \\
\frac{\beta S I}{1+\alpha_{1} S+\alpha_{2} I+\alpha_{3} S I}-(\mu+\alpha+d) I & =0, \\
\alpha I-(\mu+\gamma) R & =0 .
\end{aligned}
$$

It is clear that system (1) always has a disease-free equilibrium point $E_{0}=$ $\left(\frac{\Lambda}{\mu}, 0,0\right)$, that is, there is no infection present in the population and all individuals are susceptible. Further, if $R_{0}>1$, then system (1) has a unique endemic equilibrium $E^{*}=\left(S^{*}, I^{*}, R^{*}\right)$, where

$$
\begin{aligned}
S^{*} & =\frac{\Lambda-\varpi I^{*}}{\mu}, \\
I^{*} & =\frac{2 \rho\left(\mu+\alpha_{1} \Lambda\right)\left(R_{0}-1\right)}{\varpi\left(\beta-\alpha_{1} \rho\right)+\rho\left(\alpha_{2} \mu+\alpha_{3} \Lambda\right)+\sqrt{\Delta}}, \\
R^{*} & =\frac{\alpha}{\mu+\gamma} I^{*},
\end{aligned}
$$

with $\varpi=\frac{(\mu+\gamma)(\mu+d)+\alpha \mu}{\mu+\gamma}, \rho=\mu+\alpha+d$ and

$$
\begin{aligned}
\Delta & =\left[\varpi\left(\beta-\alpha_{1} \rho\right)+\rho\left(\alpha_{2} \mu+\alpha_{3} \Lambda\right)\right]^{2}-4 \alpha_{3} \rho \varpi\left[\beta \Lambda-\left(\mu+\alpha_{1} \Lambda\right) \rho\right] \\
& =\left[\varpi\left(\beta-\alpha_{1} \rho\right)+\rho\left(\alpha_{2} \mu-\alpha_{3} \Lambda\right)\right]^{2}+4 \alpha_{3} \mu \rho^{2}\left(\varpi+\alpha_{2} \Lambda\right) .
\end{aligned}
$$

\subsection{Stability of the disease-free equilibrium}

This subsection is devoted to studying the local stability of the disease-free equilibrium $E_{0}$ of system (1). For this, let $x(t)=S(t)-\frac{\Lambda}{\mu}, y(t)=I(t)$ and $z(t)=R(t)$. Then the linearized system of (1) around $E_{0}$ takes the following form

$$
\left\{\begin{array}{l}
D^{\theta} x(t)=-\mu x(t)-\frac{\beta \Lambda}{\mu+\alpha_{1} \Lambda} y(t)+\gamma z(t), \\
D^{\theta} y(t)=\frac{\beta \Lambda}{\mu+\alpha_{1} \Lambda} y(t-\tau)-(\mu+\alpha+d) y(t), \\
D^{\theta} z(t)=\alpha y(t)-(\mu+\gamma) z(t) .
\end{array}\right.
$$


The associated characteristic equation of system (3) can be described as

$$
\Delta(s)=\operatorname{det}\left(\begin{array}{ccc}
s^{\theta}+\mu & \frac{\beta \Lambda}{\mu+\alpha_{1} \Lambda} & -\gamma \\
0 & s^{\theta}-\frac{\beta \Lambda}{\mu+\alpha_{1} \Lambda} e^{-s \tau}+\mu+\alpha+d & 0 \\
0 & -\alpha & s^{\theta}+\mu+\gamma
\end{array}\right)=0
$$

which leads to

$$
\Delta(s)=\left(s^{\theta}+\mu\right)\left(s^{\theta}+\mu+\gamma\right)\left[s^{\theta}+(\mu+\alpha+d)\left(1-R_{0} e^{-s \tau}\right)\right]=0 .
$$

Theorem 8. If $R_{0}<1$, then the disease-free equilibrium $E_{0}$ is locally asymptotically stable for all $\tau \geq 0$.

Proof. When $\tau=0$, the coefficient matrix $M$ of system (3) satisfies

$$
M=\left(\begin{array}{ccc}
-\mu & -\frac{\beta \Lambda}{\mu+\alpha_{1} \Lambda} & \gamma \\
0 & \frac{\beta \Lambda}{\mu+\alpha_{1} \Lambda}-(\mu+\alpha+d) & 0 \\
0 & \alpha & -(\mu+\gamma)
\end{array}\right)
$$

The eigenvalues of the coefficient matrix $M$ are $\lambda_{1}=-\mu<0, \lambda_{2}=$ $-(\mu+\gamma)<0$, and $\lambda_{3}=(\mu+\alpha+d)\left(R_{0}-1\right)<0$ if $R_{0}<1$. Whence $\left|\arg \lambda_{i}\right|=\pi>\frac{\pi}{2}(i=1,2,3)$, so that all the eigenvalues $\lambda$ of $M$ satisfy $|\arg (\lambda)|>\frac{\pi}{2}$ if $R_{0}<1$.

For $\tau>0$, assume that $s=i w=w\left(\cos \frac{\pi}{2}+i \sin \frac{\pi}{2}\right)$ is a root of Eq. (4), where $w>0$. Then

$$
w^{\theta}\left(\cos \frac{\theta \pi}{2}+i \sin \frac{\theta \pi}{2}\right)+(\mu+\alpha+d)\left[1-R_{0}(\cos w \tau-i \sin w \tau)\right]=0 .
$$

Separating real and imaginary parts gives

$$
\left\{\begin{array}{l}
(\mu+\alpha+d) R_{0} \cos w \tau=w^{\theta} \cos \frac{\theta \pi}{2}+(\mu+\alpha+d) \\
(\mu+\alpha+d) R_{0} \sin w \tau=-w^{\theta} \sin \frac{\theta \pi}{2}
\end{array}\right.
$$

Thus

$$
w^{2 \theta}+2 w^{\theta}(\mu+\alpha+d) \cos \frac{\theta \pi}{2}+(\mu+\alpha+d)^{2}\left(1-R_{0}^{2}\right)=0 .
$$

Obviously $(\mu+\alpha+d) \cos \frac{\theta \pi}{2} \geq 0$ and our assumption that $R_{0}<1$, then the Eq. (5) has no positive roots. Which ensures that Eq. (4) has no purely imaginary roots if $R_{0}<1$. According to Lemma 5 , the equilibrium $E_{0}$ is locally asymptotically stable for any delay $\tau \geq 0$ if $R_{0}<1$. The proof is completed. 


\subsection{Stability of the endemic equilibrium}

In this subsection, we focus on the local stability of the endemic equilibrium of system (1) (assume that $R_{0}>1$ ). Making the transformation $x(t)=S(t)-S^{*}$, $y(t)=I(t)-I^{*}$ and $z(t)=R(t)-R^{*}$. Then by linearizing system (1) around $E^{*}$, we get the following system

$$
\left\{\begin{aligned}
D^{\theta} x(t) & =-m_{1} x(t)-m_{2} y(t)+\gamma z(t), \\
D^{\theta} y(t) & =m_{3} x(t-\tau)+m_{2} y(t-\tau)-m_{4} y(t), \\
D^{\theta} z(t) & =\alpha y(t)-m_{5} z(t),
\end{aligned}\right.
$$

where

$$
\begin{aligned}
& m_{1}=\mu+\frac{\beta I^{*}\left(1+\alpha_{2} I^{*}\right)}{\left(1+\alpha_{1} S^{*}+\alpha_{2} I^{*}+\alpha_{3} S^{*} I^{*}\right)^{2}}>0, \\
& m_{2}=\frac{\beta S^{*}\left(1+\alpha_{1} S^{*}\right)}{\left(1+\alpha_{1} S^{*}+\alpha_{2} I^{*}+\alpha_{3} S^{*} I^{*}\right)^{2}}>0, \\
& m_{3}=\frac{\beta I^{*}\left(1+\alpha_{2} I^{*}\right)}{\left(1+\alpha_{1} S^{*}+\alpha_{2} I^{*}+\alpha_{3} S^{*} I^{*}\right)^{2}}>0, \\
& m_{4}=\mu+\alpha+d>0, \\
& m_{5}=\mu+\gamma>0 .
\end{aligned}
$$

Characteristic equation which is associated with system (6) is given by

$$
\Delta(s)=\operatorname{det}\left(\begin{array}{ccc}
s^{\theta}+m_{1} & m_{2} & -\gamma \\
-m_{3} e^{-s \tau} & s^{\theta}-m_{2} e^{-s \tau}+m_{4} & 0 \\
0 & -\alpha & s^{\theta}+m_{5}
\end{array}\right)=0,
$$

which is equivalent to

$$
\Delta(s)=s^{3 \theta}+p_{2} s^{2 \theta}+p_{1} s^{\theta}+p_{0}-\left(q_{2} s^{2 \theta}+q_{1} s^{\theta}+q_{0}\right) e^{-s \tau}=0,
$$

where

$$
\begin{aligned}
p_{2} & =m_{1}+m_{4}+m_{5}>0, \\
p_{1} & =m_{1}\left(m_{4}+m_{5}\right)+m_{4} m_{5}>0, \\
p_{0} & =m_{1} m_{4} m_{5}>0, \\
q_{2} & =m_{2}>0, \\
q_{1} & =m_{2}\left(m_{1}-m_{3}+m_{5}\right)=m_{2}\left(\mu+m_{5}\right)>0, \\
q_{0} & =m_{2} m_{5}\left(m_{1}-m_{3}\right)+\alpha \gamma m_{3}=\mu m_{2} m_{5}+\alpha \gamma m_{3}>0 .
\end{aligned}
$$


Theorem 9. If $R_{0}>1$, then the endemic equilibrium $E^{*}$ is locally asymptotically stable for all $\tau \geq 0$.

Proof. When $\tau=0$, the characteristic equation of the coefficient matrix $M$ of system (6) is

$$
\lambda^{3}+\left(p_{2}-q_{2}\right) \lambda^{2}+\left(p_{1}-q_{1}\right) \lambda+p_{0}-q_{0}=0,
$$

where

$$
\begin{aligned}
& p_{2}-q_{2}=m_{1}+m_{5}+\left(m_{4}-m_{2}\right), \\
& p_{1}-q_{1}=\left(m_{1}+m_{5}\right)\left(m_{4}-m_{2}\right)+m_{1} m_{5}+m_{2} m_{3},
\end{aligned}
$$

and

$$
\begin{aligned}
p_{0}-q_{0} & =m_{1} m_{5}\left(m_{4}-m_{2}\right)+m_{2} m_{3} m_{5}-\alpha \gamma m_{3} \\
& =m_{1} m_{4} m_{5}-\mu m_{2} m_{5}-\alpha \gamma m_{3} \\
& =\mu\left(m_{4}-m_{2}\right) m_{5}+\left(m_{4} m_{5}-\alpha \gamma\right) m_{3} \\
& =\mu\left(m_{4}-m_{2}\right) m_{5}+[(\mu+\gamma)(\mu+d)+\alpha \mu] m_{3} .
\end{aligned}
$$

Note that

$$
m_{4}-m_{2}=\frac{\beta S^{*} I^{*}\left(\alpha_{2}+\alpha_{3} S^{*}\right)}{\left(1+\alpha_{1} S^{*}+\alpha_{2} I^{*}+\alpha_{3} S^{*} I^{*}\right)^{2}} \geq 0,
$$

then it is easy to show that $p_{2}-q_{2}>0, p_{1}-q_{1}>0, p_{0}-q_{0}>0$ and $\left(p_{2}-q_{2}\right)\left(p_{1}-\right.$ $\left.q_{1}\right)>p_{0}-q_{0}$. Thus by the Routh-Hurwitz criterion, all roots $\lambda_{i}(i=1,2,3)$ of (8) have negative real part, so that all the eigenvalues of $M$ of system (6) satisfy $|\arg (\lambda)|>\frac{\pi}{2}$ if $R_{0}>1$.

For $\tau>0$, let $s=i w$ be a root of Eq. (7), with $w>0$. Then

$$
\left\{\begin{array}{l}
A_{2} \cos w \tau+B_{2} \sin w \tau=A_{1}, \\
B_{2} \cos w \tau-A_{2} \sin w \tau=B_{1},
\end{array}\right.
$$

where

$$
\begin{aligned}
& A_{1}=w^{3 \theta} \cos \frac{3 \theta \pi}{2}+p_{2} w^{2 \theta} \cos \theta \pi+p_{1} w^{\theta} \cos \frac{\theta \pi}{2}+p_{0}, \\
& B_{1}=w^{3 \theta} \sin \frac{3 \theta \pi}{2}+p_{2} w^{2 \theta} \sin \theta \pi+p_{1} w^{\theta} \sin \frac{\theta \pi}{2}, \\
& A_{2}=q_{2} w^{2 \theta} \cos \theta \pi+q_{1} w^{\theta} \cos \frac{\theta \pi}{2}+q_{0},
\end{aligned}
$$




$$
B_{2}=q_{2} w^{2 \theta} \sin \theta \pi+q_{1} w^{\theta} \sin \frac{\theta \pi}{2} .
$$

Eliminating $\tau$ by squaring and adding the two equations in (10), we obtain

$$
w^{6 \theta}+\eta_{5} w^{5 \theta}+\eta_{4} w^{4 \theta}+\eta_{3} w^{3 \theta}+\eta_{2} w^{2 \theta}+\eta_{1} w^{\theta}+\eta_{0}=0,
$$

where

$$
\begin{aligned}
\eta_{5} & =2 p_{2} \cos \frac{\theta \pi}{2} \\
\eta_{4} & =p_{2}^{2}-q_{2}^{2}+2 p_{1} \cos \theta \pi \\
\eta_{3} & =2\left[\left(p_{2} p_{1}-q_{2} q_{1}\right) \cos \frac{\theta \pi}{2}+q_{0} \cos \frac{3 \theta \pi}{2}\right], \\
\eta_{2} & =p_{1}^{2}-q_{1}^{2}+2\left(p_{2} p_{0}-q_{2} q_{0}\right) \cos \theta \pi \\
\eta_{1} & =2\left(p_{1} p_{0}-q_{1} q_{0}\right) \cos \frac{\theta \pi}{2} \\
\eta_{0} & =p_{0}^{2}-q_{0}^{2} .
\end{aligned}
$$

Since $\theta \in(0,1]$ and $p_{2}>0$, then $\eta_{5} \geq 0$. In addition, we have

$$
p_{0}^{2}-q_{0}^{2}=\left(p_{0}-q_{0}\right)\left(p_{0}+q_{0}\right)>0
$$

and

$$
p_{1} p_{0}-q_{1} q_{0}=p_{1}\left(p_{0}-q_{0}\right)+q_{0}\left(p_{1}-q_{1}\right)>0,
$$

since $p_{0}-q_{0}>0, p_{1}-q_{1}>0$ and $p_{1}, p_{0}, q_{0}>0$. Then $\eta_{0}>0$ and $\eta_{1} \geq 0$.

Further, we have

$$
p_{2} p_{0}-q_{2} q_{0}=p_{2}\left(p_{0}-q_{0}\right)+q_{0}\left(p_{2}-q_{2}\right)>0,
$$

since $p_{0}-q_{0}>0, p_{2}-q_{2}>0$ and $p_{2}, q_{0}>0$, then

$$
\begin{aligned}
\eta_{2}= & p_{1}^{2}-q_{1}^{2}+2\left(p_{2} p_{0}-q_{2} q_{0}\right) \cos \theta \pi \\
\geq & p_{1}^{2}-q_{1}^{2}-2\left(p_{2} p_{0}-q_{2} q_{0}\right) \\
= & {\left[m_{1}\left(m_{4}+m_{5}\right)+m_{4} m_{5}\right]^{2}-\left[m_{2}\left(\mu+m_{5}\right)\right]^{2} } \\
& -2\left(m_{1}+m_{4}+m_{5}\right) m_{1} m_{4} m_{5}+2 m_{2}\left(\mu m_{2} m_{5}+\alpha \gamma m_{3}\right) \\
= & m_{1}^{2} m_{4}^{2}-\mu^{2} m_{2}^{2}+m_{1}^{2} m_{5}^{2}+\left(m_{4}^{2}-m_{2}^{2}\right) m_{5}^{2}+2 \alpha \gamma m_{2} m_{3} \\
= & \left(\mu^{2}+m_{5}^{2}\right)\left(m_{4}^{2}-m_{2}^{2}\right)+\left(m_{3}+2 \mu\right) m_{3} m_{4}^{2}+m_{1}^{2} m_{5}^{2}+2 \alpha \gamma m_{2} m_{3},
\end{aligned}
$$

and since $p_{1}>0$, we have

$$
\eta_{4}=p_{2}^{2}-q_{2}^{2}+2 p_{1} \cos \theta \pi
$$




$$
\begin{aligned}
& \geq p_{2}^{2}-q_{2}^{2}-2 p_{1} \\
& =\left(m_{1}+m_{4}+m_{5}\right)^{2}-m_{2}^{2}-2 m_{1}\left(m_{4}+m_{5}\right)-2 m_{4} m_{5} \\
& =m_{1}^{2}+m_{5}^{2}+m_{4}^{2}-m_{2}^{2} .
\end{aligned}
$$

From (9), we have $m_{4}^{2}-m_{2}^{2} \geq 0$ since $m_{4}+m_{2}>0$, then $\eta_{2}>0$ and $\eta_{4}>0$. On the other hand, we have

$$
\begin{aligned}
& p_{2} p_{1}-q_{2} q_{1}-3 q_{0} \\
= & \left(m_{1}+m_{4}+m_{5}\right)\left(m_{1} m_{4}+m_{1} m_{5}+m_{4} m_{5}\right)-m_{2}^{2}\left(\mu+m_{5}\right) \\
& -3 \mu m_{2} m_{5}-3 \alpha \gamma m_{3} \\
= & m_{1}^{2}\left(m_{4}+m_{5}\right)+m_{4}^{2}\left(m_{1}+m_{5}\right)+m_{5}^{2}\left(m_{1}+m_{4}\right)+3 m_{1} m_{4} m_{5} \\
& -m_{2}^{2}\left(\mu+m_{5}\right)-3 \mu m_{2} m_{5}-3 \alpha \gamma m_{3} \\
= & m_{1}^{2}\left(m_{4}+m_{5}\right)+m_{5}^{2}\left(m_{1}+m_{4}\right)+\left(\mu+m_{5}\right)\left(m_{4}^{2}-m_{2}^{2}\right)+m_{3} m_{4}^{2} \\
& +3 \mu m_{5}\left(m_{4}-m_{2}\right)+3[(\mu+\gamma)(\mu+d)+\alpha \mu] m_{3} .
\end{aligned}
$$

Thus $p_{2} p_{1}-q_{2} q_{1}>3 q_{0}$, and consequently $\eta_{3} \geq 2 q_{0}\left(3 \cos \frac{\theta \pi}{2}+\cos \frac{3 \theta \pi}{2}\right)$.

The function $\varphi: \theta \in(0,1] \mapsto 3 \cos \frac{\theta \pi}{2}+\cos \frac{3 \theta \pi}{2}$ is decreasing since for all $\theta \in(0,1]$, we have

$$
\begin{aligned}
\varphi^{\prime}(\theta) & =-\frac{3 \pi}{2}\left(\sin \frac{\theta \pi}{2}+\sin \frac{3 \theta \pi}{2}\right) \\
& =-\frac{3 \pi}{2}\left[(1+\cos \theta \pi) \sin \frac{\theta \pi}{2}+\sin \theta \pi \cos \frac{\theta \pi}{2}\right] \leq 0
\end{aligned}
$$

Then $\varphi(\theta) \geq \varphi(1)=0$, thus $\eta_{3} \geq 0$.

Therefore the Eq. (11) has no positive real roots, which ensures that Eq. (7) has no purely imaginary roots. According to Lemma 5 , the equilibrium $E^{*}$ is asymptotically stable for any delay $\tau \geq 0$ if $R_{0}>1$. The proof is completed.

\section{Conclusion}

In this paper, we have presented a delayed fractional order SIRS epidemic model with the Caputo fractional derivative and a specific functional response given by $\frac{\beta S I}{1+\alpha_{1} S+\alpha_{2} I+\alpha_{3} S I}$. We have shown that if the basic reproduction number $R_{0}$, is less than one, the disease-free equilibrium is locally asymptotically stable, which means that the disease will go to extinction. Moreover, we have proved that if $R_{0}>1$, the endemic equilibrium is locally asymptotically stable for all 
$\tau \geq 0$ and $\theta \in(0,1]$, so the disease will be persistent at the unique endemic equilibrium. From our theoretical results, we deduce that the different values of $\theta$ and $\tau$ have no effect on the stability of both equilibria. However, we don't consider the global stabilities of the two equilibria. In our future work, we investigate the global stability of fractional mathematical model (1).

\section{References}

[1] A. Korobeinikov, Lyapunov functions and global stability for SIR and SIRS epidemiologcal models with non-linear transmission, Bull. Math. Biol., 68, No 3 (2006), 615-626.

[2] G. Zaman, Y. Kang, I.H. Jung, Stability analysis and optimal vaccination of an SIR epidemic model, Biosyst., 93, No 3 (2008), 240-249.

[3] L.H. Zhou, M. Fan, Dynamics of an SIR epidemic model with limited medical resources revisited, Nonlinear Anal. Real World Appl., 13, No 1 (2012), 312-324.

[4] X.B. Liu, L.J. Yang, Stability analysis of an SEIQV epidemic model with saturated incidence rate, Nonlinear Anal. Real World Appl., 13, No 6 (2012), 2671-2679.

[5] H.F. Huo, G.M. Qiu, Stability of a mathematical model of malaria transmission with relapse, Abstract and Applied Analysis, 2014 (2014), Art. ID 289349.

[6] X. Zhang, K. Wang, Stochastic SIR model with jumps, Appl. Math. Lett., 26, No 8 (2013), 867-874.

[7] C. Zhu, G. Zeng, Y. Sun, The threshold of a stochastic SIRS model with vertical transmission and saturated incidence, Disc. Dyn. Nat. Soc., 2017 (2017), Art. ID 5620301.

[8] M. Naim, F. Lahmidi, A. Namir, Extinction and persistence of a stochastic SIS epidemic model with vertical transmission, specific functional response and Lévy jumps, Commun. Math. Biol. Neurosc., 2019 (2019), Art. ID 15.

[9] I. Podlubny, Fractional-order systems and $P I^{\lambda} D^{\mu}$ controllers, IEEE Trans. Autom. Control, 44, No 1 (1999), 208-214. 
[10] L.M. Petrovic, D.T. Spasic, T.M. Atanackovic, On a mathematical model of a human root dentin, Dental Materials, 21, No 2 (2005), 125-128.

[11] I.S. Jesus, J.A.T. Machado, J.B. Cunha, Fractional electrical impedances in botanical elements, J. of Vibration and Control, 14, No 9-10 (2008), 1389-1402.

[12] G.L. Jia, Y. X. Ming, Study on the viscoelasticity of cancellous bone based on higher-order fractional models, In: Proc. of 2nd Internat. Conf. on Bioinformatics and Biomedical Engineering, iCBBE (2008), 1733-1736.

[13] J. Korbel, Y. Luchko, Modelling of financial processes with a space-time fractional diffusion equation of varying order, Fract. Calc. Appl. Anal., 19, No 6 (2016), 1414-1433.

[14] I. Ameen, P. Novati, The solution of fractional order epidemic model by implicit Adams methods, Applied Math. Modelling, 43 (2017), 78-84.

[15] H.A.A. El-Saka, The fractional-order SIR and SIRS epidemic models with variable population size, Math. Sci. Lett., 2, No 3 (2013), 195-200.

[16] H.A.A. El-Saka, The fractional-order SIS epidemic model with variable population size, J. of the Egyptian Math. Soc., 22, No 1 (2014), 50-54.

[17] A.M. Yousef, S.M. Salman, Backward bifurcation in a fractional-order SIRS epidemic model with a nonlinear incidence rate, IJNSNS, 17, No 7-8 (2016), 401-412.

[18] D. Rostamy, E. Mottaghi, Forward and backward Bifurcation in a fractional-order SIR epidemic model with vaccination, Iranian J. of Science \& Technology, 42, No 2 (2018), 663-671.

[19] X. Wang, Z. Wang, X. Huang, Y. Li, Dynamic analysis of a fractional-order delayed SIR model with saturated incidence and treatment functions, Int. J. Bifurc. Chaos, 28, No 14 (2018), 1850180.

[20] K. Hattaf, N. Yousfi, A. Tridane, Stability analysis of a virus dynamics model with general incidence rate and two delays, Appl. Math. Comput., 221 (2013), 514-521.

[21] I. Podlubny, Fractional Differential Equations, Academic Press, San Diego etc. (1999). 
[22] I. Petras, Fractional-Order Nonlinear Systems: Modeling, Analysis and Simulation, Springer (2011).

[23] W. Deng, C. Li, J. Lu, Stability analysis of linear fractional differential system with multiple time delays, Nonlinear Dyn., 48, No 4 (2007), 409416.

[24] H. Wang, Y. Yu, G. Wen, S. Zhang, Stability analysis of fractional-order neural networks with time delay, Neural Process. Lett., 42, No 2 (2015), 479-500.

[25] O. Diekmann, J.A.P. Heesterbeek, Mathematical Epidemiology of Infectious Diseases, John Wiley \& Sons, New York (2000). 
